\title{
Rotary resonance in multiple-quantum magic-angle spinning
}

\author{
Zhehong Gan ${ }^{\text {a,* }}$, Philip Grandinetti ${ }^{\mathrm{b}}$ \\ ${ }^{a}$ Center of Interdisciplinary Magnetic Resonance, National High Magnetic Field Laboratory, Tallahassee, FL 32310, USA \\ b Department of Chemistry, Ohio State University, Columbus, OH 43210-1173, USA
}

Received 30 October 2001; in final form 22 November 2001

\begin{abstract}
The theory of rotary resonance in multiple-quantum magic-angle spinning (MQMAS) experiment is presented. The rotary resonance effect can enhance the efficiency of MQMAS experiment for high-resolution NMR spectra of half-integer quadrupolar nuclei [J. Chem. Phys. 114 (2001) 4618]. Using a spin-1/2 formalism and Floquet theorem, the spin dynamics of a spin-3/2 under $\mathrm{rf}$ irradiation and magic-angle sample spinning is solved analytically in a doubly rotating frame. The results show an oscillatory behavior of MQ excitation with nulls at $\omega_{1}=n \omega_{\mathrm{r}} / 2$, where $\omega_{1}$ represents the rf field strength and $\omega_{\mathrm{r}}$ is the spinning frequency. Efficient MQ excitation occurs between these nulls and MQ conversion peaks at $\omega_{1}=n \omega_{\mathrm{r}}$. The origin of the rotary resonance phenomenon is related to frequency shift of a general two-level system under a randomly modulated periodic perturbation. (C) 2002 Elsevier Science B.V. All rights reserved.
\end{abstract}

\section{Introduction}

Recently Vosegaard et al. [1] have discovered a rotary resonant phenomenon in exciting and converting multiple-quantum (MQ) coherence of half-integer quadrupolar nuclei. Under fast magic-angle sample spinning, the excitations of MQ coherence can be greatly enhanced when spinning frequency $\omega_{\mathrm{r}}$ and $\mathrm{rf}$ field strength $\omega_{1}$ are between adjacent rotary resonance conditions $\omega_{1}=n \omega_{\mathrm{r}} / 2$. The conversion of MQ coherence to central transitions can be enhanced with $\omega_{1}=n \omega_{\mathrm{r}}$. The discovery of this rotary resonance effect has lead to the FAster Spinning gives Transfer Enhancement at Rotary resonance (FASTER) experiment that improves the efficiency of multiple-quantum magic-angle spinning (MQMAS) experiment to obtain highresolution NMR spectra of half-integer quadrupolar nuclei [1]. The FASTER experiment has several advantages compared to other MQMAS pulse schemes [2-8]: it avoids interferences between fast sample spinning and $\mathrm{rf}$ irradiation that other pulse schemes may have [9] and it requires low $\omega_{1}$ making it particularly suitable for low- $\gamma$ nuclei.

\footnotetext{
${ }^{*}$ Corresponding author. Fax: +1-850-644-1366.

E-mail address: gan@magnet.fsu.edu (Z. Gan).
} 
The rotary resonance effect was first discovered by a numerical study followed immediately by experimental demonstration [1]. The origin of the resonance effect, however, has not yet been fully understood because of the complexity of the spin dynamics. For quadrupolar nuclei, the spin dynamics under rf irradiation is complicated for two reasons: first, nuclei with spin $>1 / 2$ have more than two energy-levels making the problem of a time-dependent Hamiltonian difficult to solve; second, quadrupolar interactions usually are two-orders of magnitude larger than typical spinning frequencies. Modulation of large quadrupolar couplings by sample rotation makes the application of average Hamiltonian theory difficult even at high-spinning speed.

In this Letter we present a theoretical study of the MQMAS rotary resonance phenomenon. First the multilevel system of a model spin-3/2 is separated into two two-level subsystems represented by spin-1/2 Hamiltonians. Floquet theory is then applied in a doubly rotating frame solving the time evolution of the spin-1/2 Hamiltonians. The combination of spin-1/2 formalism and Floquet theorem leads to an effective Hamiltonian that provides a complete analytical solution to the spin dynamics of quadrupolar nuclei. As the rotary resonance phenomenon has already been studied thoroughly by numerical simulations, this Letter focuses on the theoretical aspect especially the origin of the resonance phenomenon. It will be shown that the rotary resonance is related to a randomly modulated periodic perturbation in a general two-level system.

\section{Spin-1/2 formalism}

We consider a Hamiltonian that includes only the first-order quadrupolar interaction and the rf spin interaction

$$
H(t)=q(t)\left(3 S_{z}^{2}-S^{2}\right) / 6+\omega_{1} S_{x} .
$$

The quadrupolar coupling frequency $q(t)$ is modulated by sample rotation. The phase and amplitude of the rf irradiation are assumed constant. Time evolution of an observable $Q$ follows:

$$
\langle Q\rangle=\operatorname{Tr}\left[Q \cdot U(t) \sigma(0) U(t)^{-1}\right]
$$

where $\sigma(0)$ is the initial density operator and $U(t)$ is the time propagator of the Hamiltonian. The density and observable operators for two-dimensional MQMAS experiment are $\sigma(0)=3 T_{z}+C_{z}, Q=T_{y}$ for MQ excitation and $\sigma(0)=T_{+}, Q=C_{-}$for MQ conversion. Here $T$ and $C$ represent the triple-quantum and central transition spin operators, respectively.

For a spin-3/2, the four-level Hamiltonian can be separated into two two-level subsystems through a rotation

$$
R=\frac{1}{\sqrt{2}}\left(\begin{array}{llll}
1 & 0 & 0 & 1 \\
0 & 1 & 1 & 0 \\
0 & 1 & -1 & 0 \\
1 & 0 & 0 & -1
\end{array}\right)
$$

that mixes pairs of base states with opposite signs $(|m\rangle \pm|-m\rangle) / \sqrt{2}[10,11]$

$$
H=\frac{1}{2}\left(\begin{array}{llll}
q & \sqrt{3} \omega_{1} & 0 & 0 \\
\sqrt{3} \omega_{1} & -q+2 \omega_{1} & 0 & 0 \\
0 & 0 & -q-2 \omega_{1} & \sqrt{3} \omega_{1} \\
0 & 0 & \sqrt{3} \omega_{1} & q
\end{array}\right)=\left[\begin{array}{ll}
H_{a} & 0 \\
0 & H_{b}
\end{array}\right] .
$$

The two-level subsystems can be described independently by spin-1/2 operators $I_{x}, I_{y}, I_{z}$ and 


$$
E=\frac{1}{2}\left(\begin{array}{ll}
1 & 0 \\
0 & 1
\end{array}\right)
$$

(see e.g. [12-14]),

$$
\begin{aligned}
& U(t)=\left[\begin{array}{ll}
U_{a}(t) & 0 \\
0 & U_{b}(t)
\end{array}\right], \\
& H_{a}(t)=\omega_{1} E+\left[-\omega_{1}+q(t)\right] I_{z}+\sqrt{3} \omega_{1} I_{x}, \\
& H_{b}(t)=-\omega_{1} E+\left[-\omega_{1}-q(t)\right] I_{z}+\sqrt{3} \omega_{1} I_{x} .
\end{aligned}
$$

The spin-1/2 Hamiltonians are transferred further into a doubly rotating frame. The first rotation $R_{q}=\mathrm{e}^{\mp \mathrm{i} \varphi(t) I_{z}}, \varphi(t)=\int_{0}^{t} q\left(t^{\prime}\right) \mathrm{d} t^{\prime}$ eliminates the large quadrupolar coupling term $q(t)$. The $\mp$ signs are for $H_{a}$ and $H_{b}$, respectively. The second rotation $R_{\mathrm{rf}}=\mathrm{e}^{\mathrm{Fin} \omega_{\mathrm{r}} t / 2} \cdot \mathrm{e}^{\mathrm{i} n \omega_{\mathrm{r}} t_{z}}$ reduces $\omega_{1}$ to a rotary resonance offset $\Delta \omega_{1}=\omega_{1}-n \omega_{\mathrm{r}}$ with $n$ the closest integer to $\omega_{1} / \omega_{\mathrm{r}}$

$$
\begin{aligned}
& H_{a}(t)=\Delta \omega_{1}\left(E-I_{z}\right)+\frac{\sqrt{3}}{2} \omega_{1}\left[I_{+} \mathrm{e}^{-\mathrm{i} \varphi(t)-\mathrm{i} n \omega_{\mathrm{r}} t}+I_{-} \mathrm{e}^{\mathrm{i} \varphi(t)+\mathrm{i} n \omega_{\mathrm{r}} t}\right], \\
& H_{b}(t)=-\Delta \omega_{1}\left(E+I_{z}\right)+\frac{\sqrt{3}}{2} \omega_{1}\left[I_{+} \mathrm{e}^{\mathrm{i} \varphi(t)-\mathrm{i} n \omega_{\mathrm{r}} t}+I_{-} \mathrm{e}^{-\mathrm{i} \varphi(t)+\mathrm{i} n \omega_{\mathrm{r}} t}\right] .
\end{aligned}
$$

Both rotations keep the same periodicity of the Hamiltonians. The phase modulation by quadrupolar coupling can be expanded in a Fourier series

$$
\mathrm{e}^{\mathrm{i} \varphi(t)}=\sum_{k=-\infty}^{\infty} S_{k} \mathrm{e}^{\mathrm{i} k \omega_{\mathrm{r}} t}
$$

The expansion coefficient $S_{k}$ is the individual intensity contribution to the $k$ th spinning sideband under MAS of the first-order quadrupolar interaction.

The rotating frame and basis rotation change matrix representations of operators $A \Rightarrow R \cdot A \cdot R^{-1}$. The rotation by the quadrupolar Hamiltonian commutes with all central and triple-quantum transition operators therefore does not affect the observable and density operators relevant to MQMAS experiment. The basis change rotates the indices of spin operators $x, y, z$ and the rf rotation modulates additionally the central transition operators:

$$
\begin{aligned}
& T_{x}=S_{z}^{1,4}, \quad C_{x}=S_{z}^{2,3}, \\
& T_{y}=-S_{y}^{1,4}, \quad C_{y}=\mathrm{i}\left(S_{+}^{2,3} \mathrm{e}^{-\mathrm{i} 2 n \omega_{\mathrm{r}} t}-S_{-}^{2,3} \mathrm{e}^{\mathrm{i} 2 n \omega_{\mathrm{r}} t}\right) / 2, \\
& T_{z}=S_{x}^{1,4}, \quad C_{z}=\left(S_{+}^{2,3} \mathrm{e}^{-\mathrm{i} 2 n \omega_{\mathrm{r}} t}+S_{-}^{2,3} \mathrm{e}^{\mathrm{i} 2 n \omega_{\mathrm{r}} t}\right) / 2 .
\end{aligned}
$$

The spin dynamics of MQ excitation and conversion can be obtained explicitly in terms of the spin-1/2 propagator elements in the doubly rotating frame:

$$
\begin{aligned}
& S_{\mathrm{ex}}(t)=\operatorname{Tr}\left[T_{y} \cdot U(t)\left(3 T_{z}+C_{z}\right) U^{-1}(t)\right]=\operatorname{Im}\left[\frac{3}{2} U_{\alpha \alpha}^{a} U_{\beta \beta}^{b *}-\frac{1}{2} U_{\alpha \beta}^{a} U_{\alpha \beta}^{b} \mathrm{e}^{-\mathrm{i} 2 n \omega_{\mathrm{r}} t}\right], \\
& S_{\text {con }}(t)=\operatorname{Tr}\left[T_{+} \cdot U(t) C_{-} U(t)^{-1}\right]=\frac{\left|U_{\alpha \beta}^{a}\right|^{2}+\left|U_{\alpha \beta}^{b}\right|^{2}}{4}-\frac{\operatorname{Re}\left[U_{\alpha \beta}^{a} U_{\alpha \beta}^{b} \mathrm{e}^{-\mathrm{i} 2 n \omega_{\mathrm{r}} t}\right]}{2},
\end{aligned}
$$

where $\alpha$ and $\beta$ represent two states of a spin-1/2. In $S_{\mathrm{ex}}(t)$, the first and the second term are the excitation from the polarization of triple-quantum $T_{z}$ and central transitions $C_{z}$, respectively. In the conversion, the first and the second term are the in-phase and out-phase coherence transfer with respect to the rf pulse. 
With the spin-1/2 formalism, the spin dynamics of the quadrupolar nuclei has been transformed into solving the propagators of the spin-1/2 Hamiltonians in the doubly rotating frame that will be described in the following section.

\section{Floquet theory}

For a time-dependent Hamiltonian, an analytical solution of the propagator is not generally available even with a simple two-level system. Floquet theory can transfer the problem of a periodic Hamiltonian into a time-independent one. Numerous applications of Floquet theory can be found in solid state NMR of rotating samples [15-21]. In this section, the formalism of Floquet theory is briefly described followed by a treatment to the spin-1/2 Hamiltonians.

Floquet theorem starts by separating the propagator into a periodic part $P(t)$ and a constant Hamiltonian $Q[22]$

$$
U(t)=P(t) \mathrm{e}^{-\mathrm{i} Q t} P(0)^{-1} .
$$

The periodic operator $P(t)$ is expanded in a Fourier series and so is the Hamiltonian

$$
P(t)=\sum_{k=-\infty}^{\infty} P^{(k)} \mathrm{e}^{\mathrm{i} k \omega_{\mathrm{r}} t}, \quad H(t)=\sum_{k=-\infty}^{\infty} H^{(k)} \mathrm{e}^{\mathrm{i} k \omega_{\mathrm{r}} t} .
$$

The expansion coefficient $P^{(k)}$ and $Q$ satisfy an equation directly derived from the Schrödinger equation

$$
\sum_{k=-\infty}^{\infty}\left(H^{(l-k)}+l \omega_{\mathrm{r}} \delta_{k l}\right) P^{(k)}=P^{(l)} Q .
$$

Thus $Q$ and $P^{(k)}$ can be obtained as the eigenvalues and eigenvectors of a so-called Floquet Hamiltonian

$$
H^{F}=\sum_{k, l=-\infty}^{\infty}|l\rangle\left(H^{(l-k)}+l \omega_{\mathrm{r}} \delta_{k l}\right)\langle k| .
$$

The Floquet Hamiltonian is time-independent but its matrix spans infinitely in Floquet space. The matrix is often truncated because of the increasing diagonal elements with the Floquet index.

The Floquet matrix of the spin-1/2 Hamiltonian can be obtained explicitly by replacing their elements with the corresponding Floquet sub-matrices:

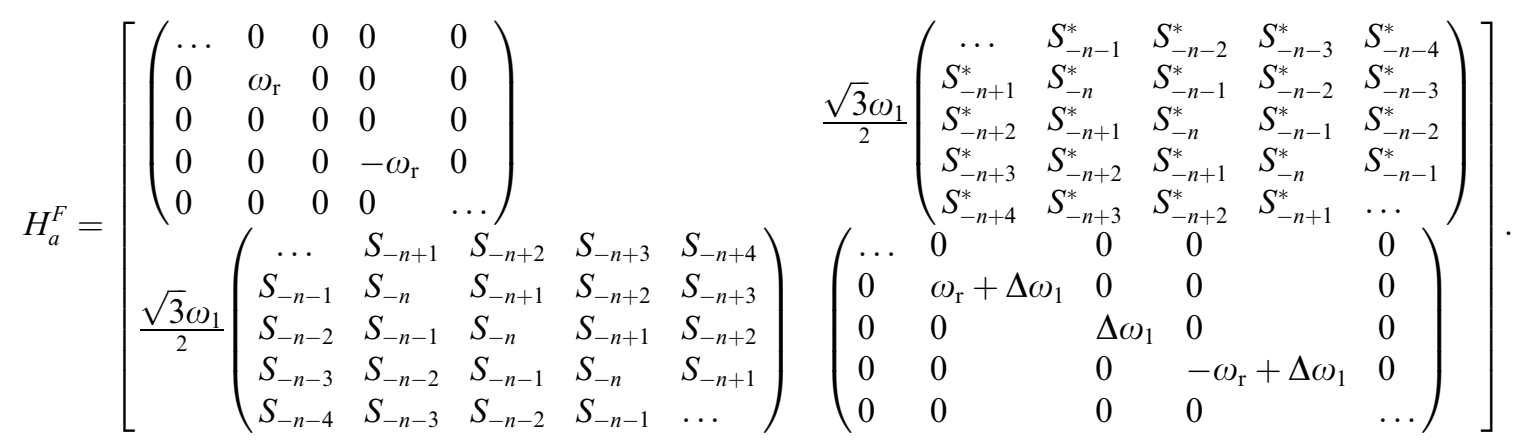

Off-diagonal elements are non-zero only between $\alpha$ and $\beta$. Under fast MAS and moderate rf field strength, these elements are usually small compared to $\omega_{\mathrm{r}}$ due to the scaling by spinning sideband intensity $S_{k}$. The 
sum of sideband intensities is normalized $\sum\left|S_{k}\right|^{2}=1$ and there are many sidebands by large first-order quadrupolar interactions.

The eigenvalues and eigenvectors of the Floquet matrix can be obtained by reducing off-diagonal elements through Jacobi transformations [23]. Jacobi transformation is a rotation that eliminates one pair of off-diagonal elements at a time:

$$
\begin{aligned}
& J_{i j}^{-1}\left(\begin{array}{ll}
E_{i} & V_{i j} \\
V_{i j}^{*} & E_{j}
\end{array}\right) J_{i j}=\left(\begin{array}{cl}
E_{i}+\Delta / 2 & 0 \\
0 & E_{j}-\Delta / 2
\end{array}\right), \\
& J_{i j}=\left(\begin{array}{cc}
1 & \Delta / 2 V_{i j} \\
-\Delta / 2 V_{i j}^{*} & 1
\end{array}\right), \\
& \Delta=-\left(E_{i}-E_{j}\right)+s_{E_{i}-E_{j}} \sqrt{\left(E_{i}-E_{j}\right)^{2}+4\left|V_{i j}\right|^{2}},
\end{aligned}
$$

where $s_{E_{i}-E_{j}}$ is the sign of $E_{i}-E_{j}$. It is worthy mentioning that $J_{i j}$ is slightly different from [23] by a scaling factor. Successive Jacobi transformations through all off-diagonal elements reduce the Floquet matrix to nearly diagonal despite that individual Jacobi rotation may cause a little undo to previously annihilated elements. The overall rotation matrix of the successive Jacobi transformations $\Pi J_{i j}$ can be approximated as

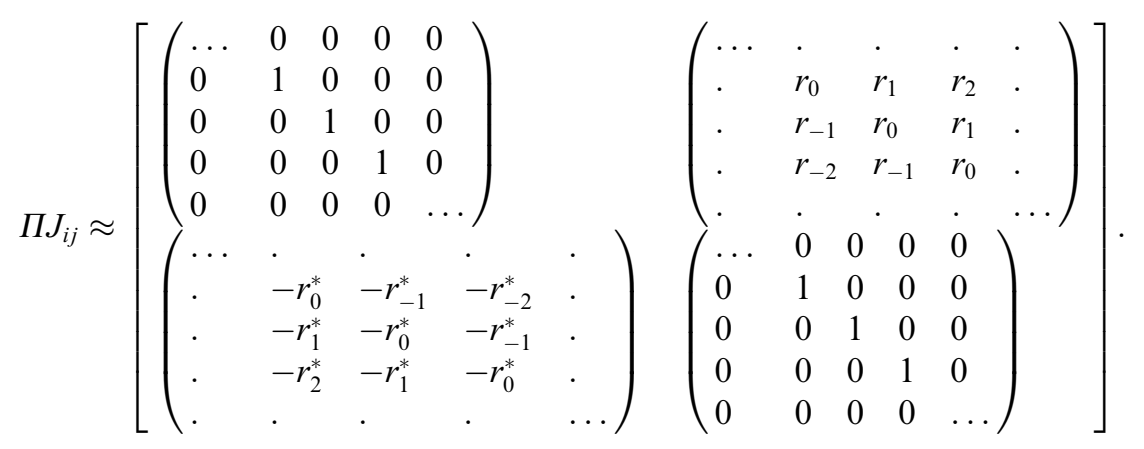

The resulting shifts of diagonal elements of the Hamiltonian and the rotation matrix elements are

$$
\begin{aligned}
& \Delta \omega_{a, b}=\sum_{k} \Delta_{k}^{a, b}, \\
& \Delta_{k}^{a, b}=-\left(k \omega_{\mathrm{r}}+\Delta \omega_{1}\right)+s_{k+\Delta \omega_{1} / \omega_{\mathrm{r}}} \sqrt{\left(k \omega_{\mathrm{r}}+\Delta \omega_{1}\right)^{2}+3 \omega_{1}^{2}\left|S_{\mp(n+k)}\right|^{2}}, \\
& r_{k}^{a}=\frac{\Delta_{k}^{a}}{\sqrt{3} \omega_{1} S_{-(n+k)}^{*}}, \quad r_{k}^{b}=\frac{\Delta_{k}^{b}}{\sqrt{3} \omega_{1} S_{n+k}} .
\end{aligned}
$$

The index $a, b$ and $\mp$ sign are for $H_{a}^{F}$ and $H_{b}^{F}$, respectively. It is important to note that one set of eigenvalue and eigenvector is sufficient for each Floquet sub-matrix because the eigenvalues of Floquet Hamiltonian are separated by the modulation frequency with the corresponding eigenvector elements shifted by the Floquet index.

The $Q$ and $P^{(k)}$ operators can be obtained from the eigenvalue and the eigenvector extracted from the rotation matrix $\Pi J_{i j}$ :

$$
\begin{aligned}
Q_{a, b} & = \pm \Delta \omega_{1} E+\left(\Delta \omega_{a, b}-\Delta \omega_{1}\right) I_{z}, \\
P_{a, b}^{(k)} & =\left(\begin{array}{ll}
\delta_{k 0} & r_{k}^{a, b} \\
-r_{k}^{a, b *} & \delta_{k 0}
\end{array}\right),
\end{aligned}
$$


where $\delta_{k 0}$ is the discrete $\delta$-function. The results lead to an analytical expression of the spin-1/2 propagator:

$$
U(t)=\sum_{k} P^{(k)} \mathrm{e}^{-\mathrm{i} Q t+\mathrm{i} k \omega_{\mathrm{r}} t} P(0)^{-1},
$$

$P(0)=\sum P^{(k)}$ is an unitary matrix and can be generally described by a spin- $1 / 2$ rotation operator with Euler angles $(\varphi, \theta,-\varphi)[24]$ :

$$
\begin{aligned}
& P(0)=\sum_{k} P^{(k)}=\cos ^{-1} \frac{\theta}{2} R, \quad R=\mathrm{e}^{-\mathrm{i} I_{z} \varphi} \mathrm{e}^{-\mathrm{i} I_{y} \theta} \mathrm{e}^{\mathrm{i} I_{z} \varphi}, \\
& \tan \frac{\theta}{2} \mathrm{e}^{-\mathrm{i} \varphi}=-\sum_{k} r_{k}^{a, b} .
\end{aligned}
$$

When $t=n \tau_{\mathrm{r}}$, the propagator can be described by an effective Hamiltonian because $P\left(n \tau_{\mathrm{r}}\right)=P(0)$

$$
\begin{aligned}
& U\left(n \tau_{\mathrm{r}}\right)=P(0) \mathrm{e}^{-\mathrm{i} Q t} P(0)^{-1}=\mathrm{e}^{-\mathrm{i} H_{\text {eff }} t}, \\
& H_{\text {eff }}=R Q R^{-1} .
\end{aligned}
$$

This simplification is also valid in the case that rotor modulation of spin dynamics and spinning sidebands in frequency domain are negligible. The full propagator and effective Hamiltonian in the doubly rotating frame are given by

$$
\begin{aligned}
& U(t)=\left[\begin{array}{cl}
\mathrm{e}^{-\mathrm{i}\left(\vec{\omega}_{a}^{\text {eff }} \cdot \vec{I}+\Delta \omega_{1} E\right) t} & 0 \\
0 & \mathrm{e}^{-\mathrm{i}\left(\vec{\omega}_{b}^{\mathrm{eff}} \cdot \vec{I}-\Delta \omega_{1} E\right) t}
\end{array}\right] \\
& H_{\mathrm{eff}}=\left[\begin{array}{cl}
\vec{\omega}_{a}^{\mathrm{eff}} \cdot \vec{I}+\Delta \omega_{1} E & 0 \\
0 & \vec{\omega}_{b}^{\mathrm{eff}} \cdot \vec{I}-\Delta \omega_{1} E
\end{array}\right] .
\end{aligned}
$$

The Hamiltonian is described by effective fields oriented at the polar angles $\left(\theta_{a, b}, \varphi_{a, b}\right)$

$$
\omega_{a, b}^{\mathrm{eff}}=\Delta \omega_{a, b}-\Delta \omega_{1} .
$$

The effective fields along with the frequency shift $\Delta \omega_{a, b}$ and polar angle $\left(\theta_{a, b}, \varphi_{a, b}\right)$ in Eqs. (16) and (19) summarize the results of Floquet treatment to the spin-1/2 Hamiltonians. The effective Hamiltonian can also be obtained principally by an average Hamiltonian series. Because $H_{a}(t)$ and $H_{b}(t)$ in the doubly rotating frame contain numerous modulation terms, the average Hamiltonian approach requires highorder terms to fully explain the rotary resonance phenomenon. The derivation of high-order average Hamiltonian can become very complex where as the Floquet approach provide a single analytical expression for the effective Hamiltonian despite that the Floquet formalism appeared to be complex in the beginning.

\section{Rotary resonance}

The spin dynamics of MQ excitation and conversion can be obtained explicitly in terms of the spin-1/2 propagators. We use the derived effective Hamiltonian to simplify the discussions of rotary resonance. We also focus on only the terms that are responsible for the rotary resonance effect: the excitation from triplequantum polarization $T_{z}$ and the in-phase MQ coherence transfer: 


$$
\begin{aligned}
& S_{\text {ex }}^{M Q}(t)=\operatorname{Im} \frac{3}{2}\left[\mathrm{e}^{-\mathrm{i} \Delta \omega_{1} t}\left(\cos \frac{\omega_{a}^{\mathrm{eff}} t}{2}-\mathrm{i} \cos \theta_{a} \sin \frac{\omega_{a}^{\mathrm{eff}} t}{2}\right)\left(\cos \frac{\omega_{b}^{\mathrm{eff}} t}{2}-\mathrm{i} \cos \theta_{b} \sin \frac{\omega_{b}^{\mathrm{eff}} t}{2}\right)\right], \\
& S_{\text {con }}^{\text {in-phase }}(t)=\frac{1}{4}\left[\sin ^{2} \theta_{a} \sin ^{2} \frac{\omega_{a}^{\mathrm{eff}} t}{2}+\sin ^{2} \theta_{b} \sin ^{2} \frac{\omega_{b}^{\mathrm{eff}} t}{2}\right] .
\end{aligned}
$$

Fig. 1 plots the MQ excitation and conversion curves obtained from the theory in comparisons with stepwise numerical calculations. The theoretical results show nearly perfect fits with the numerical simulations especially at low rf fields. In Floquet treatment, the eigenvalue and eigenvector were obtained from successive Jacobi transformations only once through the Floquet matrix. As off-diagonal elements are proportional to $\omega_{1}$, the deviation by this approximation is expected to increase with the rf field.

In the doubly rotating frame, the effective Hamiltonian has the following $S_{z}^{1,4}$ component:

$$
\omega_{z}^{1,4}=\Delta \omega_{1}\left(\sin ^{2} \frac{\theta_{a}}{2}+\sin ^{2} \frac{\theta_{b}}{2}\right)+\frac{\Delta \omega_{a} \cos \theta_{a}+\Delta \omega_{b} \cos \theta_{b}}{2} .
$$

For the triple-quantum transition, $\left(S_{x}^{1,4}, S_{y}^{1,4}, S_{z}^{1,4}\right)$ forms an orthogonal basis of the Liouville space: $S_{x}^{1,4}=T_{z}$ is the triple-quantum polarization; $S_{y}^{1,4}=-T_{y}$ and $S_{z}^{1,4}=T_{x}$ are imaginary and real part of the triplequantum coherence, respectively. Therefore $\omega_{z}^{1,4}$ is the triple-quantum Hamiltonian element that rotates triple-quantum polarization into the imaginary part triple-quantum coherence.

Fig. 1 shows an oscillatory curve for MQ excitation with nulls near $\omega_{1}=n \omega_{\mathrm{r}} / 2$. MQ conversion peaks at $\omega_{1}=n \omega_{\mathrm{r}}$. The modulation in MQ conversion can be explained by the polar angle alone. At off-resonance $\Delta \omega_{1} \gg \sqrt{3} \omega_{1}\left|S_{ \pm n}\right|$, the polar angles of the effective fields are small because $r_{k} \ll 1$. When on-resonance $\Delta \omega_{1} \approx 0$, the polar angles are close to $90^{\circ}$ because $r_{0}=1$. The angular factors $\sin ^{2} \theta_{a, b}$ yield peaks for MQ conversion at rotary resonance condition $\omega_{1}=n \omega_{\mathrm{r}}$. The polar angle curve $\left\langle\theta_{a, b}\right\rangle$ plotted in Fig. 1b illustrates the close relation between MQ conversion and the polar angles of the effective fields.
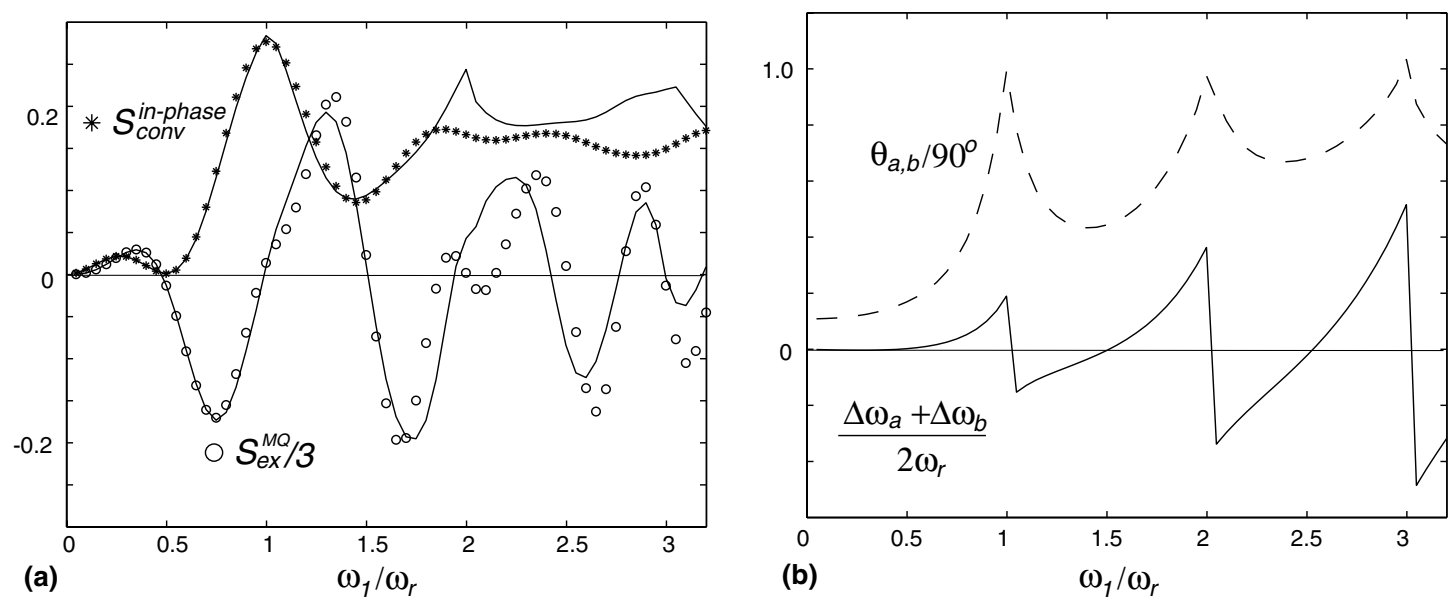

Fig. 1. (a) MQ excitation (o) and MQ conversion (*), (b) mean frequency shift $F=\left(\Delta \omega_{a}+\Delta \omega_{b}\right) / 2 \omega_{\mathrm{r}}$ and polar angle $\theta_{a, b}$ of effective fields as a function of $\mathrm{rf}$ field $\omega_{1}$ over spinning frequency $\omega_{\mathrm{r}}$. In (a), the stars and circles are from exact numerical simulations and the solid lines are the results from Floquet theory after powder averages. Quadrupolar coupling parameters $q_{\mathrm{c}}=3.2 \mathrm{MHz}, \eta=0.21$ of ${ }^{87} \mathrm{RbClO}_{4}$ were used in the calculation. The pulse length is two rotor periods and the spinning frequency is $40 \mathrm{kHz}$. 
The resonance behavior in MQ excitation can be explained as following. When on-resonance, the triplequantum Hamiltonian component $\omega_{z}^{1,4}$ is near zero because $\Delta \omega_{1} \approx 0$ and $\theta_{a, b} \approx 90^{\circ}$. At off-resonance, the triple-quantum component becomes $\omega_{z}^{1,4} \approx\left(\Delta \omega_{a}+\Delta \omega_{b}\right) / 2$ because $\theta_{a, b} \approx 0^{\circ}$. Fig. $1 \mathrm{~b}$ shows that the mean frequency shift $\left\langle\Delta \omega_{a}+\Delta \omega_{b}\right\rangle / 2$ vanishes when the rotary resonance offset equals a half of the spinning frequency $\Delta \omega_{1}=\omega_{\mathrm{r}} / 2$ (the physical insight of the vanishing frequency shift will be discussed later). Thus, the MQ excitation is at nulls for both $\omega_{1} \approx n \omega_{\mathrm{r}}$ and $\omega_{1} \approx(n+1 / 2) \omega_{\mathrm{r}}$.

The overall MQ excitation for powder samples $\left\langle\omega_{z}^{1,4}\right\rangle$ can be estimated as the following. The intensities of low-order spinning sidebands are approximately equal $S_{k} \approx \sqrt{\omega_{\mathrm{r}} / 2 \omega_{q}}$, where $2 \omega_{q}$ is the full first-order quadrupolar splitting. For simplicity, we also assume that the frequency shift $\Delta \omega_{a, b}$ and the polar angles $\theta_{a, b}$ of the effective fields can be powder-averaged separately:

$$
\begin{aligned}
& \frac{\left\langle\Delta \omega_{a}+\Delta \omega_{b}\right\rangle}{2}=F \cdot \omega_{\mathrm{r}},\left\langle\tan \frac{\theta_{a, b}}{2}\right\rangle=\frac{F}{R} \\
& F=\sum_{k=-\infty}^{\infty} s_{k+\Delta \omega_{1} / \omega_{\mathrm{r}}} \sqrt{\left(k+\Delta \omega_{1} / \omega_{\mathrm{r}}\right)^{2}+R^{2}}-\left(k+\Delta \omega_{1} / \omega_{\mathrm{r}}\right),
\end{aligned}
$$

where $F$ is a dimensionless function shown in Fig. 1 that depends on the rotary resonance offset $\Delta \omega_{1} / \omega_{\mathrm{r}}$. $R=\sqrt{3 \omega_{1}^{2} / \omega_{q} \omega_{\mathrm{r}}}$ is a parameter similar to the adiabatic parameter $\alpha=\omega_{1}^{2} / \omega_{q} \omega_{\mathrm{r}}$ introduced by Vega [11]. With this assumption, the average triple-quantum Hamiltonian can be obtained:

$$
\left\langle\omega_{z}^{1,4}\right\rangle \approx \frac{R^{2}-F^{2}+2 F \Delta \omega_{1} / \omega_{\mathrm{r}}}{R^{2}+F^{2}} F \omega_{\mathrm{r}}
$$

The expression reveals that MQ excitation is directly proportional to the mean frequency shift $\left\langle\Delta \omega_{a}+\Delta \omega_{b}\right\rangle / 2=F \cdot \omega_{\mathrm{r}}$ with nulls at $\omega_{1} \approx n \omega_{\mathrm{r}} / 2$. In Fig. 1, the excitation curve does not follow exactly the spikes of the frequency shifts near $\omega_{1}=n \omega_{\mathrm{r}}$. When $\Delta \omega_{1} \approx 0$, it can be shown that $F \approx R$ and the numerator in $\left\langle\omega_{z}^{1,4}\right\rangle$ reduces the mean triple-quantum element of the Hamiltonian. This reduction shifts the maximum MQ excitation toward the middle between adjacent nulls. This reduction can also be understood through the scaling $\cos \theta_{a, b} \approx 0$ in Eq. (24) when $\Delta \omega_{1} \approx 0$.

The rotary resonance in MQ excitation is related to the frequency shift $\Delta \omega_{a, b}$ caused by the modulating $\mathrm{rf}$ Hamiltonian in the doubly rotating frame. The spin-1/2 Hamiltonians represents a general two-level system under a randomly modulated periodic perturbation. Such a perturbation can be descried by equally spaced sidebands in the spectral density (Fig. 2) similar to the low-order spinning sidebands caused by the firstorder quadrupolar coupling where the powder average mimics the assemble average of the random modulation. Each sideband causes a frequency shift through a Bloch-Siegert type effect [25]. The overall shift is a sum from all sidebands mostly from the ones with frequency $n \omega_{\mathrm{r}}$ close to the transition frequency $\omega_{1}$. Considering the symmetry of sidebands below and above the transition frequency, the net frequency shift becomes zero when $\omega_{1}$ is exactly on one of the sidebands $\omega_{1}=n \omega_{\mathrm{r}}$ or in the middle between two adjacent sidebands $\omega_{1}=(n+1 / 2) \omega_{\mathrm{r}}$. The vanishing frequency shift at these conditions leads to nulls in MQ excitation. This picture gives a simple explanation to the origin of the rotary resonance phenomenon based on symmetry arguments.

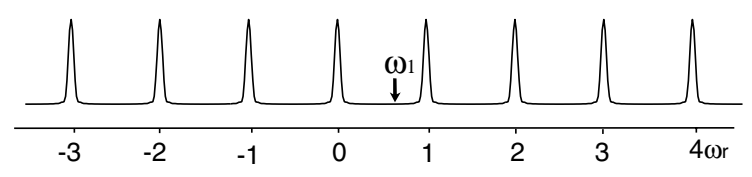

Fig. 2. Spectral density of two-level system $\left(\Delta E=\omega_{1}\right)$ under a randomly phase modulated periodic perturbation. The shift of transition frequency becomes zero when the transition frequency is on one of the sidebands or in the middle between sidebands. 


\section{Conclusions}

It has been shown that the spin dynamics of a spin-3/2 under rf irradiation and magic-angle spinning can be solved analytically using spin-1/2 formalism and Floquet theory. An effective Hamiltonian has been obtained in a doubly rotating frame. The theory explains the rotary resonance effect especially the origin of the resonance conditions. The theory can also be applied to spin dynamics of other important phenomenon such as nutation [10] and spin-lock [11] of quadrupolar nuclei under magic-angle sample spinning.

The rotary resonance phenomenon is directly related to a general problem of a two-level system under a randomly phase modulated perturbation. Based on symmetry arguments, the frequency shift from the periodic perturbation becomes zero when the transition frequency matches an integer or a half integer of the modulation frequency. The rotary resonance phenomenon in MQMAS is just one example of such a resonant behavior in frequency shift that may also occur in spin dynamics of other type of spin interactions under sample spinning.

It should be noted that an alternative theoretical treatment of this problem using bimodal Floquet theory was recently presented by J.D. Walls, K.-H. Lim and A. Pines at the 2nd Alpine Meeting on SolidState NMR in Chamonix, France.

\section{Acknowledgements}

The authors would like to thank Parthasarathy Srinivasan and Prof. J.R. Quine of the Department of Mathematics at FSU for discussions and Prof. A. Pines of UC-Berkeley for a manuscript of bimodal Floquet theory prior to publication. The work is supported by the National High Magnetic Field Laboratory through National Science Foundation Cooperative Agreement DMR0084173 and CHE0111109 to P.G.

\section{References}

[1] T. Vosegaard, P. Florian, D. Massiot, P.J. Grandinetti, J. Chem. Phys. 114 (2001) 4618.

[2] L. Frydman, J.S. Harwood, J. Am. Chem. Soc. 117 (1995) 5367.

[3] A. Medek, J.S. Harwood, L. Frydman, J. Am. Chem. Soc. 117 (1995) 12779.

[4] P.K. Madhu, A. Goldbourt, L. Frydman, S. Vega, Chem. Phys. Lett. 307 (1999) 41.

[5] P.K. Madhu, A. Goldbourt, L. Frydman, S. Vega, J. Chem. Phys. 112 (2000) 2377.

[6] A.P.M. Kentgens, R. Verhagen, Chem. Phys. Lett. 300 (1999) 435.

[7] G. Wu, D. Rovnyak, P.C. Huang, R.G. Griffin, Chem. Phys. Lett. 277 (1997) 79.

[8] T. Vosegaard, D. Massiot, P.J. Grandinetti, Chem. Phys. Lett. 326 (2000) 454.

[9] J.P. Amoureux, M. Pruski, D.P. Lang, C. Fernandez, J. Magn. Reson. 131 (1998) 170.

[10] A. Samoson, E. Lippmaa, J. Magn. Reson. 79 (1988) 255.

[11] A.J. Vega, J. Magn. Reson. 96 (1992) 50.

[12] A. Wokaun, R.R. Ernst, Chem. Phys. Lett. 52 (1977) 407.

[13] S. Vega, A. Pines, J. Chem. Phys. 66 (1977) 5624.

[14] S. Vega, J. Chem. Phys. 68 (1978) 5518.

[15] E.T. Olejniczak, J.E. Roberts, S. Vega, R.G. Griffin, J. Magn. Reson. 56 (1984) 156.

[16] A. Schmidt, S.O. Smith, D.P. Raleigh, J.E. Roberts, R.G. Griffin, S. Vega, J. Chem. Phys. 85 (1986) 4248.

[17] A. Schmidt, S. Vega, J. Chem. Phys. 87 (1987) 6895.

[18] A. Schmidt, S. Vega, J. Chem. Phys. 96 (1992) 2655.

[19] T. Nakai, C.A. Mcdowell, J. Chem. Phys. 96 (1992) 3452.

[20] S.W. Ding, C.A. McDowell, Chem. Phys. Lett. 288 (1998) 230.

[21] T.O. Levante, M. Baldus, B.H. Meier, R.R. Ernst, Mol. Phys. 86 (1995) 1195. 
[22] H.J. Shirley, Phys. Rev. B 138 (1965) 979.

[23] W.H. Press, Numerical Recipes in C: The Art of Scientific Computing, Cambridge University Press, Cambridge, 1992. [24] J.J. Sakurai, S.F. Tuan, Modern Quantum Mechanics, Benjamin/Cummings, Menlo Park, CA, 1985.

[25] F. Bloch, A. Siegert, Phys. Rev. 57 (1940) 522. 